UCRL-9B254

PREPRINT

UCRL_-98254

DE88 006831

\title{
PROPERTIES OF HYDROGEN/HELIUM ACCRETION PLASMAS
}

\author{
Charles 0. Dermer \\ Lawrence Livermore National Laboratory \\ Livermore. CA 94550
}
This paper was prepared for submittal to the Conference on Nuclear Spectroscopy of
Astrophysica? Sources
Washington, D.C.
December 14-16, 1987

February 1988

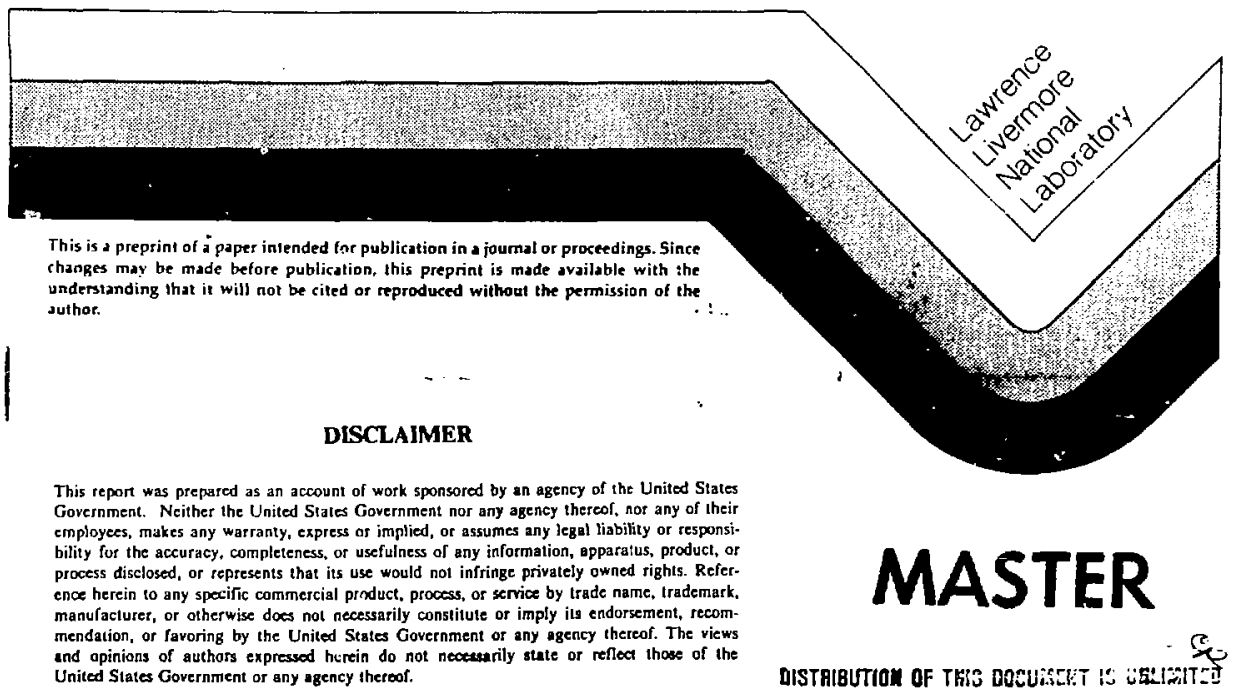




\title{
PROPERTIES OF HYDROGEN/ HELIUM ACCRETION PLASMAS
}

\author{
Nidisal Guessoum \\ Depariment of Physics, University of California, San Diego \\ La Jolla, California 92093 \\ Charies D. Dermer \\ Physics Department, Lawrence Livermore National Laboratory \\ P.O. Box 808, L-297, Livernore, Califorria 94550
}

\begin{abstract}
We study the properties of impulsively-heated plasmas initially composed of hydrogen and helium. We follow the time-dependent behavior of the ion and electron temperatures. the pair density, and the densities of hydrogen, helium, and nuclei formed in fusion and . breakup reactions. We also consider neutron production and escape, and calculate the 0.431 and $0.478 \mathrm{MeV}$ line luminosities from $\alpha-\alpha$ fusion reactions, and the $2.22 \mathrm{MeV}$ line luminosity from neutron capture on protons.
\end{abstract}

\section{RTRODUCTION}

High-temperane ion plasmas may be produced in the accretion flow near a compact object, or through shock heating. If the ion temperature $T_{i}$ exceeds $-5 \mathrm{MeV}$, as is commonly assumed (e.g., Eilek 1980; Hogan and Applegate 1987), nucleosynthetic fusion and breakup reactions will occur. The breakup reactions limit the nuclear deexcitation line lurninosity from elements such as C, N and O (Aharonian and Sunyaev 1984; Gould 1986; Guessoum, these proceedings), and provide a sourre of neutrons which, being electrically neutral, can escape from the plasma. If the only process available for exchanging energy between the ions and electrons is elastic Coulomb scattering, the electron temperature $T_{e} \lesssim$ few MeV < $\mathrm{T}_{\mathrm{i}}$ (Shapiro, Lightman and Eardley 1976; Higdon and Lingenfelter 1977).

In this paper, we investigate the processes responsible for the formation and destruction of the light isotopes $\mathrm{d},{ }^{3} \mathrm{H}$ and ${ }^{3} \mathrm{He}$ in a plasma originally composed solely of hydrogen and helium. We treat neutron production, capture, and escape, and evaluate the line emissivities from $\alpha-\alpha$ fusion reactions and from neutron capture on protons. For the parameters considered here, the abundances of the light isotopes are maximized when the initial ion temperature is $\sim 15 \mathrm{MeV}$. The luminosity of the 0.431 and $0.478 \mathrm{MeV}$ lines is found to be $\Sigma$ $10^{-5}$ of the continuum luminosity radiated by the electrons. Using a source size characteristic of a $10 \mathrm{M}_{\mathrm{o}}$ galactic black hole, we find that most neutrons formed in the breakup of 
$\alpha$-paricles leave the hot ion plasma. Consequently, the $2.2 \mathrm{MeV}$ line luminosity in the ion plasma is small, but a narrow $2.2 \mathrm{MeV}$ line could be produced if a significant fraction of the escaping neurrons are captured by protons in the atmosphere of a companion star.

\section{ANALYSIS}

We model the system by a sphere of hot plasma with radius $R=10^{7} \mathrm{~cm}$. Protons and $\alpha$-particles with densiry $n_{p}$ and $n_{\alpha}$, respectively, are impulsively heated to temperature $T_{i, 0}$ at initial time. Assuming equality of the proton and ion temperatures, vee calculate the ime-dependent behavior of $\mathrm{T}_{e}, \mathrm{~T}_{\mathrm{i}}$, the isotopic compositions, and the emiuted spectrum. We inploy roughly solar composition for the initial $\alpha$-particle densiry $n_{\alpha, 0}$, leting $n_{\alpha, 0}=$ $0 . n_{p, 0^{*}}$ The magnecic field is set equal to zero in the present work.

For the ion-electron Coulomb energy exchange rate, we use equation (11) of Dermer (1986), valid for nonrelativistic protons or ions and electrons ar arbitrary temperatures. The Coulomb logarithm $\Lambda$ is set equal to 20 . The electrons lose energy by emitting bremsstrahlung and by Comptonizing soft photons. We follow the treatment of Dermer (1988) for calculating the pair densiry and spectra resulting from the thermal Comptonization of bremsstrahlung and annihilation radiation (see also Guilbert and Stepney 1985). However, we now employ accurate fits to the exact thermal bremsstrahlung and annihilation source spectra. The low density of multi-MeV photons renders photonuclear breakup reactions unimportant compared to nuelear breakup reactions in this system, and are therefore neglected (see Guessoum, 1988).

In order to achieve an accuracy in our calculations of $-10 \%$, we need to take into account reactions involving protons, neutrons and $\alpha$-particles with each other and with the light isotopes $d,{ }^{3} \mathrm{H}$ and ${ }^{3} \mathrm{He}$. Reactions of the light isotopes with themselves can be neglected as they affect results at the $1 \%$ level or less. We also treat the production and breakup of ${ }^{6} \mathrm{Li}$, ${ }^{7} \mathrm{Li}$ and ${ }^{7} \mathrm{Be}$, which are formed in $\alpha-\alpha$ fusion reactions, and are accompanied by the emission of gamma-ray lines at 0.431 and $0.478 \mathrm{MeV}$. We have derived the thermal-avernged reaction rates for these reactions, and will present the detailed results in subsequent work (Guessoum and Dermer 1988). A typical rate has the form

$$
r_{12}=n_{1} n_{2} A_{12} \theta^{s} \exp (-\alpha / \theta) g(\theta),
$$

where $A_{12}$ and $s$ are constants, $n_{j}$ is the density of particle $j, \theta=T_{j} / m_{e} c^{2}$, and $g(\theta)$ is a polynomial function. The constant $a$ is proportional to the threshold energy of the reaction. This form follows from the basic expression for the reaction rate at nonrelativistic temperatures (see Guessoum, these proceedings). For example, the important breakup 
reaction $\mathrm{p}+\alpha \rightarrow{ }^{3} \mathrm{He}+\mathrm{d}$ has a rate given by the expression

$$
r=n_{p} n_{a} 2.0 \times 10^{-15} \theta^{-1 / 2} \exp (-35.9 / \theta)(1+0.025 \theta)
$$

The form of equation (1) also holds for breakup reactions involving incident neutrons. But because neutrons are electrically neutral, they will not be confined by electromagtietic fields and may leave the system. The fraction of neutrons that freely escape from a spherical volume in time $\delta t$ is $\sim \delta t v\left(T_{j}\right) / R$, where the average neutron velocity $v\left(T_{i}\right) \sim\left(3 T_{i} / m_{i 1}\right)^{1 / 2}$. The escape of seutrons is impeded by neutron-proton and, to a lesser extent, neutron-ion collisions. We generalize the fractional neutron escape rate by letting $R \rightarrow R\left(1+\tau_{p n} / \beta\right)$, where $\tau_{p n}=n_{p} \sigma_{p n}\left(E_{n}\right) R$ and $E_{n}=T_{i}$. For the neutron-proton cross section $\sigma_{p n}$, we use the fit of Gammel (1963). Neutron decay is accounted for by a loss term employing the neutron decay lifetime. Time-dilation effects to the neutron lifetime are small and can be negelected. We also neglect gravitational effects to neutron escape (cf. Aharonian and Suryaev 1984).

\section{RESULTS}

In Fig. 1 we show the time-variation of the electron and ion temperatures following the impulsive heating of protons and $\alpha$-particles to temperature $T_{i, 0}=10 \mathrm{MeV}$. In this simulation, the density of the injected protons comesponds to unit optical depth of the system, that is, $n_{p} \sigma_{T} R=\tau_{p}=1$, implying that the initial proton density $n_{p}=1.5 \times 10^{17} \mathrm{~cm}^{-3}$. The pair density $\ll n_{p}$ throughout the simulation. As can be seen, $T_{e}$ hovers at $-0.5 \mathrm{MeV}$ during the 100 or so Thomson times that it takes the ions to transfer their energy to the electrons. The average luminosity radiated by the electrons during this period is $-3 \times 10^{35} \mathrm{erg} / \mathrm{s}$. (In general, the average bolometric luminosity $\propto$ $T_{i, o} \tau_{p}{ }^{2} R$ for nonmagnetic systems with $T_{i, 0} \lesssim 50 \mathrm{MeV}$; pair effects increase the average luminosity at higher initial ion

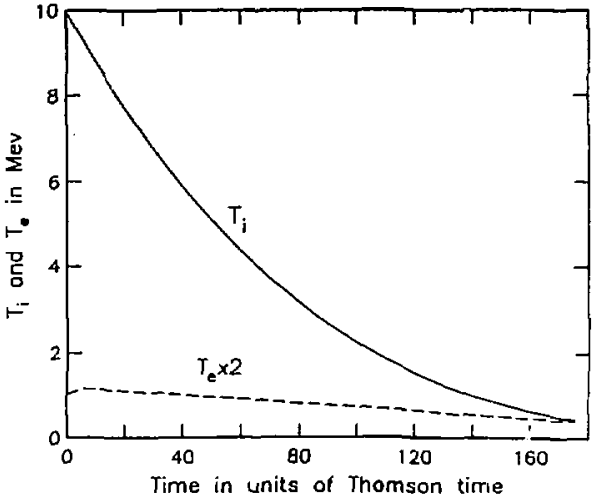

Fig. 1. Time-variation of the electron and ion temperatures as a function of time in units of Thomson time $\mathrm{t}_{\mathrm{T}}=\left(\mathrm{n}_{\mathrm{p}, 0} \sigma_{\mathrm{T}} \mathrm{c}\right)^{-1}=3.3 \times 10^{-4} \mathrm{sec}$. The initial ion temperature is $10 \mathrm{MeV}$. 
temperatures [Guilbert and Stepney 1985].) In Fig. 2 we show the time-variation of the abundances of the various isotopes. Due to nuclear breakup reactions, there is a depletion of the $\alpha$-particle abundance that appears in the form of $n, d,{ }^{3} H$ and ${ }^{3} \mathrm{He}$. After reaching a peak value, the abundances of the light isotopes declines somewhat due to the increasing importance of light element breakup compared to helium breakup.

The breakup of $\alpha$-particles is dominated by the reaction $p+\alpha$ $\rightarrow{ }^{3} \mathrm{He}+\mathrm{d}$. The abundance of ${ }^{3} \mathrm{He}$ at late time is much greater than that of ${ }^{3} \mathrm{H}$, which is primarily formed in the less important reaction $\mathrm{p}+\alpha \rightarrow{ }^{3} \mathrm{H}+$ $\mathrm{p}+\mathrm{p}$. The ${ }^{3} \mathrm{He}$ abundance exceeds that of $d$, partly because d has a lower nuclear binding energy and is therefore more rapidly broken down at low $(-1$ $\mathrm{MeV}$ ) ion temperatures than ${ }^{3} \mathrm{He}$. The final abundances of the various isotopes as a function of initial ion temperature are shown in Fig. 3 for the case $\tau_{p}=$ 1. At higher values of $T_{i, 0}, \mathbf{a}$ decreasing fraction of $\alpha$-particles survives to low temperature. Indeed, matter that has been processed through a high (>15 $\mathrm{MeV}$ ) ion-temperature state may show ${ }^{3} \mathrm{He}$ and $\mathrm{d}$ abundances

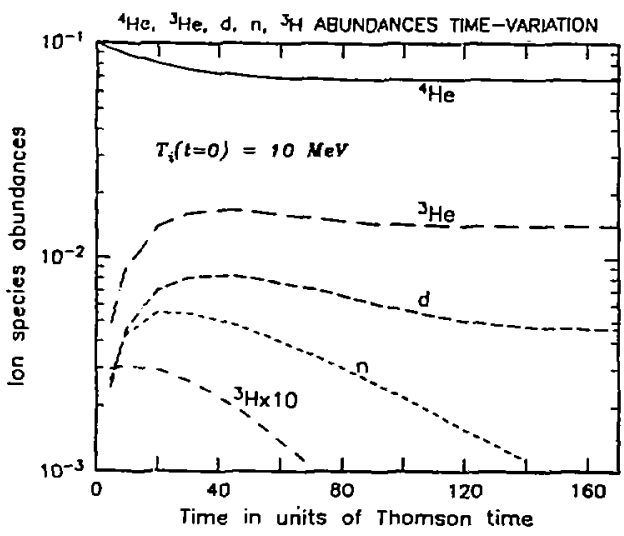

Fig. 2. Time-variation of ratios of the neutron, a-paricie, $4,{ }^{3} \mathrm{H}$ and ${ }^{3} \mathrm{He}$ densities to the proton density as a function of time in units of Thomson time. The initial ion temperature is $10 \mathrm{MeV}$.

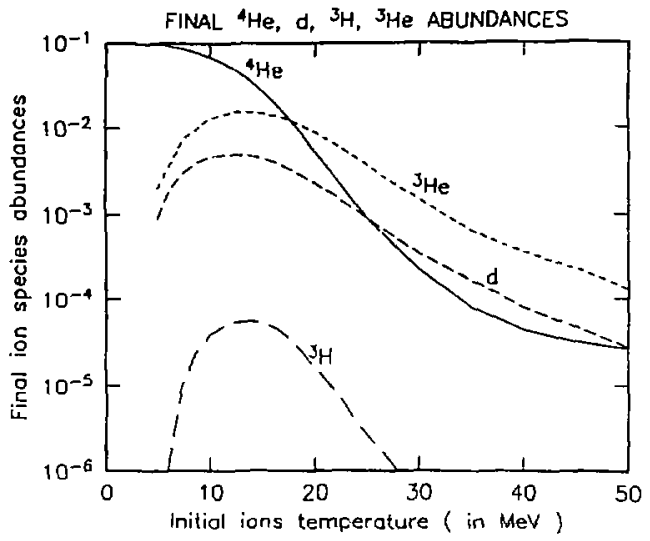

Fig. 3. Final $\alpha$-particle, d, ${ }^{3} \mathrm{H}$ and ${ }^{3} \mathrm{He}$ aburdances of the plasma following the impulsive heating of $\mathrm{H}$ and $\mathrm{He}$ $\omega$ various initial ion temperatures. 
which are comparable to or exceed the final $\alpha$-particle abundance.

Time-averaged spectra emitted by this system are typical Comptonized thermal bremsstrahlung spectra, characterized by the form $E^{-q_{e x p}}\left(-E /\left\langle T_{e}\right\rangle\right)$, where the photon spectral index $q<1$ and $\left\langle\mathrm{T}_{\mathrm{e}}>\right.$ is an effective electron temperature. Deexcitation of ${ }^{7} \mathrm{Li}^{*}$ and ${ }^{7} \mathrm{Be}^{*}$ which are produced in the reactions $\alpha+\alpha \rightarrow{ }^{7} \mathrm{Li}^{*}+p$ and $\alpha+\alpha \rightarrow{ }^{7} \mathrm{Be}^{*}+n$ yields gamma-ray lines at 0.478 and $0.431 \mathrm{MeV}$, respectively. The other important line is at 2.224 MeV from the neutron capture reaction $n+p \rightarrow d+\gamma$. A measure of the line strength is determined by the ratio of the line luminosity to the continuum luminosity, the latter being equal to the energy transferred from the ions to the electrons through Coulomb coupling. In Table 1 we show the relative line and continuum luminosities as a function of $T_{i, 0^{*}}$ The relative line luminosity initially increases with $T_{i, 0}$ as the thermal reaction barrier is overcome. At higher $\mathrm{T}_{\mathrm{i}, \mathrm{o}}$, the reaction rate approaches a constant value, and the relative line luminosity decreases as the ion the:mal energy available for the formation of the continuum increases. The ratio of line luminosity to continuum luminosity for the $\alpha-\alpha$ fusion lines is $\lesssim$ $10^{-5}$, and would be hidden by the continuum unless $<T_{e}>\ll 0.4 \mathrm{MeV}$ or $n_{\alpha} \approx n_{p}$. The relative $2.224 \mathrm{MeV}$ line luminosity in the source is likewise small since most neutrons escape rather than form reuterium through neutron capture on protons. This is apparent from Fig. 2, where one sees that although the composition of the isotopes stabilizes at late times, the neutron abundance continues to decline due to escape. In addition, the maximum ratio of the neutron to proton density is only -0.0055 , whereas a value of 0.037 would be expected if the neutrons remain in the source (Table 1).

TABLE I

RELATIVE LINE LUMINOSITIES FROM AND NEUTRON PRODUCTION IN HOT ION PLASMA

\begin{tabular}{|c|c|c|c|c|}
\hline $\mathrm{T}_{\mathrm{i}, \mathrm{o}}(\mathrm{MeV})$ & $\frac{\mathrm{L}_{0,431+0,478}}{\varepsilon_{p \rightarrow c}}$ & $\frac{L_{2 n 4}}{E_{p \rightarrow 0}}$ & $\frac{N_{n}}{N_{p}}$ & $\frac{\phi_{224}^{(a)}}{f_{224}}$ \\
\hline 5.0 & $2.0 \times 10^{-6}$ & $2.7 \times 10^{-7}$ & $1.7 \times 10^{-3}$ & $2.8 \times 10^{-6}$ \\
\hline 10.0 & $1.0 \times 10^{-5}$ & $1.2 \times 10^{-6}$ & $3.7 \times 10^{-2}$ & $3.1 \times 10^{-5}$ \\
\hline 20.0 & $5.3 \times 10^{-6}$ & $8.6 \times 10^{-7}$ & 0.17 & $7.2 \times 10^{-5}$ \\
\hline 30.0 & $1.7 \times 10^{-6}$ & $29 \times 10^{-7}$ & 0.197 & $5.5 \times 10^{-5}$ \\
\hline 40.0 & $7.8 \times 10^{-7}$ & $1.4 \times 10^{-7}$ & 0.198 & $4.2 \times 10^{-5}$ \\
\hline 50.0 & $4.8 \times 10^{-7}$ & $9.1 \times 10^{-8}$ & 0.199 & $3.4 \times 10^{-5}$ \\
\hline
\end{tabular}

(a) Estimated $2.224 \mathrm{MeV}$ line fux from neutron capture on procons in the companion of Cygnus X-1. 
A line signature at $2.224 \mathrm{MeV}$ could be formed, however, if the neutrons leaving the hot ion plasma are captured on a companion star. In the case of Cyg X-1, Coulomb coupling by hot ions at $T_{i} \sim 50 \mathrm{MeV}$ could provide the heating source of the electrons and pairs which produce the continuum luminesity (Liang and Dermer 1988). Scaling to values appropriate to Cyg X-1, we have estimated the $2.224 \mathrm{MeV}$ line flux expected if some fraction of the neutrons are captured by protons in the armosphere of the companion star HDE226868. The results are listed in Table 1 , using $2.5 \mathrm{kpe}$ for the distance to Cyg X-1. The factor $\mathrm{f}_{2.224}$ represents the racio of $2.224 \mathrm{MeV}$ photons escaping from the companion star to the number of neutrons leaving the hot ion plasma. Observation of a narrow $2.224 \mathrm{MeV}$ line at the level of $-10^{-6}-10^{-5}$ photons $\mathrm{cm}^{-2}-\mathrm{s}^{-1}$ from Cyg X-1 would provide strong support for a two-temperanre plasma model, but may requre the next generation of gamma-ray telescopes.

The work of C. D. was performed under the auspices of the U.S. D.O.E. by the Lawrence Livermore National Laboratory under contract number W-7405-ENG-48.

\section{REFERENCES}

Aharonian, F.A, and Sunyaev, R.A. 1984, MNRAS, 210, 257.

Dermer, C.D. 1986, Ap. J., $307,47$.

Dermer, C.D. 1988, in Georgia State Universiry Conference on Active Galactic Nuclei, eds.

H.R Miller and P.J. Wiita (Springer-Verlag), in press.

Eiiek, J. A. 1980, Ap. J., 236, 664.

Gammel, J. 1963, Fast Neurron Physics, par I, eds. J.B. Marion and J.L. Fowler

(Interscience Publishers: New York), p. 2209.

Gould, R.J. 1986, Nuclear Phys., B266, 737.

Guessoum, N. 1988, in preparacion.

Guessoum, N. and Dermer, C.D. 1988, in preparacion.

Guilbert, P.W. and Stepney, S. 1985, MNRAS, 212, 187.

Higdon, J.C. and Lingenfelter, RE. 1977, Ap. J., 215, L53.

Hogan, C. J. and Applegate, J. H. 1987, Nature, 330, 236.

Liang, E.P. and Denmer, C.D. 1988, Ap. J. (Leners), 325, in press.

Slıapiro, S.L.., Lightman, A.P., and Eardley, D.M. 1976, Ap.J., 2J4, 187. 